\title{
In vitro platelet adhesion on polymeric surfaces with varying fluxes of continuous nitric oxide release
}

\author{
Yiduo Wu, Zhengrong Zhou, Mark E. Meyerhoff \\ Department of Chemistry, The University of Michigan, Ann Arbor, Michigan 48109-1055
}

Received 6 April 2006; revised 8 August 2006; accepted 19 September 2006

Published online 24 January 2007 in Wiley InterScience (www.interscience.wiley.com). DOI: 10.1002/jbm.a.31105

\begin{abstract}
Nitric oxide (NO) is released by endothelial cells that line the inner walls of healthy blood vessels at fluxes ranging from $0.5 \times 10^{-10}$ to $4.0 \times 10^{-10} \mathrm{~mol} \mathrm{~cm}^{-2}$ $\min ^{-1}$, and this continuous NO release contributes to the extraordinary thromboresistance of the intact endothelium. To improve the biocompatibility of blood-contacting devices, a biomimetic approach to release/generate $\mathrm{NO}$ at polymer/blood interfaces has been pursued recently (with NO donors or NO generating catalysts doped within polymeric coatings) and this concept has been shown to be effective in preventing platelet adhesion/activation via several in vivo animal studies. However, there are no reports to date describing any quantitative in vitro assay to evaluate the blood compatibilities of such NO release/generating polymers with controlled NO fluxes. Such a methodology is desired to provide a preliminary assessment of any new NO-releasing material, in terms of the effective-
\end{abstract}

ness of given $\mathrm{NO}$ fluxes and NO donor amounts on platelet activity before the more complex and costly in vivo testing is carried out. In this article, we report the use of a lactate dehydrogenase assay to study in vitro platelet adhesion on such NO-releasing polymer surfaces with varying NO fluxes. Reduced platelet adhesion was found to correlate with increasing NO fluxes. The highest NO flux tested, $7.05( \pm 0.25) \times 10^{-10} \mathrm{~mol} \mathrm{~cm}{ }^{-2} \mathrm{~min}^{-1}$, effectively reduced platelet adhesion to nearly $20 \%$ of its original level (from $14.0( \pm 2.1) \times 10^{5}$ cells $\mathrm{cm}^{-2}$ to $2.96( \pm 0.18)$ $\times 10^{5}$ cells $\mathrm{cm}^{-2}$ ) compared to the control polymer coating without NO release capability. (C) 2007 Wiley Periodicals, Inc. J Biomed Mater Res 81A: 956-963, 2007

Key words: nitric oxide; blood-compatibility; platelet adhesion; lactate dehydrogenase assay; plasticized poly(vinyl chloride)

\section{INTRODUCTION}

Nitric oxide (NO) is now widely recognized as a potent inhibitor of platelet adhesion and activation, ${ }^{1,2}$ as well as a naturally occurring vasodilator. ${ }^{3}$ In fact, the extraordinarily thromboresistant nature of blood vessel inner walls is, in part, due to the continuous production of $\mathrm{NO}$ by the endothelial cells that line such vessels. ${ }^{4,5}$ The NO flux from the endothelium is estimated to be in the range of $0.5 \times 10^{-10}$ to $4.0 \times$ $10^{-10} \mathrm{~mol} \mathrm{~cm}^{-2} \mathrm{~min}^{-1}$. Previous studies based on the addition of discrete NO donors into blood samples suggested that higher NO levels produced from such donors exert greater antiplatelet activities. ${ }^{6-8}$ However, these experiments failed to simulate the function of a healthy endothelium, which produces a low but continuous flux of $\mathrm{NO}$ from its surface. One report by Ramamurthi and Lewis using flowing platelets in a

Correspondence to: M.E. Meyerhoff; e-mail: mmeyerho@ umich.edu

Contract grant sponsor: National Institutes of Health; contract grant numbers: EB-000783, HD-015434

(C) 2007 Wiley Periodicals, Inc. membrane-based diffusion device showed that platelet adhesion to a collagen-coated membrane (using radiolabeled platelets) was inhibited by very low fluxes of NO (much less than physiological fluxes) but the effect leveled off rapidly as increasing NO fluxes were used by changing gas phase NO levels on the opposite side of the membrane device. ${ }^{9}$ Research in this $\mathrm{lab}^{10-20}$ and elsewhere ${ }^{21,22}$ has been aimed at preparing more biocompatible polymeric coatings that continuously produce NO at physiological levels to mimic the function of the healthy endothelium, thus creating nonthrombogenic surfaces. Indeed, a growing number of reports have already demonstrated the improved blood compatibility of NO-releasing polymeric materials using in vivo animal model studies. ${ }^{10-15}$ It has also been reported that discrete additions of $\mathrm{NO}$ or its donor species (e.g., diazeniumdiolates, nitrosothiols, etc.) can inhibit platelet adhesion and activation in vitro. ${ }^{6-8,23-25}$ However, there is lack of a simple and reliable in vitro method to assay the platelet adhesion on polymers that continuously release $\mathrm{NO}$, and to assess how varying levels of NO release effect platelet adhesion. Such a method would be attractive to carry out preliminary biocompatibility studies of any new 
NO release/generating polymers, before undertaking more expensive in vivo testing.

The two most commonly used in vitro platelet adhesion assays are scanning electron microscopy $(\mathrm{SEM})^{26}$ and radioisotope-labeled platelet counting. ${ }^{27}$ However, both methods have their own drawbacks that render them unsuitable for quantitative assay. SEM only studies a very small fraction of the total surface that had been in contact with platelets, of which the "representativeness" is always questionable. Radiolabeling, on the other hand, suffers from the potential for preactivation of platelet as a result of the labeling step.

In recent years, the assay of lactate dehydrogenase (LDH) present in platelets has been reported to provide a useful approach to study in vitro platelet adhesion on chemically and/or physically modified surfaces. ${ }^{28-32} \mathrm{LDH}$ is normally stored within intact platelets and other blood cells. Elevated serum LDH levels are usually associated with cytolytic events. ${ }^{33}$ When platelets adhered to polymer surfaces are lysed using a surfactant solution, the amount of $\mathrm{LDH}$ released into the bulk solution is proportional to the number of cells adhered. The activity of LDH can be measured by a simple colorimetric assay and used to quantify platelet adhesion. In this article, we report, for the first time, the use of an $\mathrm{LDH}$ assay to study the efficacy of varying levels of NO, a bioactive species, released from poly(vinyl chloride) (PVC) films doped with a lipophilic diazeniumdiolate type $\mathrm{NO}$ donor, in reducing platelet adhesion onto the surface of such polymeric materials. It will be shown that increasing fluxes of NO decrease the observed platelet adhesion.

\section{MATERIALS AND METHODS}

\section{Preparation of NO-releasing polymer coatings}

Poly(vinyl chloride) (PVC) (average $M_{\mathrm{W}}=106,000$, Aldrich, Milwaukee, WI) was mixed with sebacic acid di(2ethylhexyl) ester (DOS, Sigma, St. Louis, MO) in dry tetrahydrofuran (Fisher, Pittsburgh, PA) to make a solution containing $2 \%(\mathrm{w} / \mathrm{v})$ PVC and $2 \%(\mathrm{w} / \mathrm{v})$ DOS. For control films, $N, N^{\prime}$-dibutyl-1,6-hexanediamine (DBHD, Aldrich) was mixed with potassium tetrakis(4-chlorophenyl)-borate (KTpClPB, Fluka, Buchs, Switzerland) in a 1:1 molar ratio and dispersed in the polymer solution to yield $4 \mathrm{wt} \%$ of DBHD in the final cured polymer film. The NO adduct, $\mathrm{DBHD} / \mathrm{N}_{2} \mathrm{O}_{2}$ (diazeniumdiolated DBHD), was synthesized as previously reported. ${ }^{10}$ DBHD $/ \mathrm{N}_{2} \mathrm{O}_{2}$ and equal molar KTpCIPB were dispersed into the polymer casting solution in a similar manner as the control film to yield 0.50, 1.0, 2.0, and $4.0 \mathrm{wt} \%$ of final DBHD $/ \mathrm{N}_{2} \mathrm{O}_{2}$ loadings. The coating cocktails were sonicated for $15 \mathrm{~min}$ and then $0.25 \mathrm{~mL}$ aliquots were placed in wells of a 96-well round-bottom polypropylene microtiterplates (Evergreen Scientific, Los Angeles, CA).
The polymer films were allowed to cure under ambient condition overnight before $3 \mu \mathrm{m}$ layers of plain PVC/DOS (1:1) were applied as top coats. The resulting polymer films were allowed to cure for another $24 \mathrm{~h}$ before use.

\section{NO-release measurement}

NO released from the different polymer-coated wells was measured via a chemiluminescence NO Analyzer ${ }^{\mathrm{TM}}$, Model 280 (Sievers Instruments, Boulder, CO), to assess the NO-release profile and to determine the optimal time window for platelet adhesion studies. The entire polymercoated well was immersed in phosphate buffered saline (PBS, $137 \mathrm{mM} \mathrm{NaCl}, 2.7 \mathrm{mM} \mathrm{KCl}, 10 \mathrm{mM}$ sodium phosphate, $\mathrm{pH}$ 7.4) at $37^{\circ} \mathrm{C}$ and the solution was bubbled with $\mathrm{N}_{2}$. NO released from the polymer coating within the well was purged from the buffer, carried by the $\mathrm{N}_{2}$ gas into the chemiluminescence reaction chamber of the instrument, and monitored in real-time.

\section{Platelet adhesion studies}

Arterial blood from New Zealand white rabbits, weighing 2.5-3 kg, was drawn into 9:1 volume of blood:anticoagulant citrate/phosphate/dextrose solution (Abbott Labs, North Chicago, IL) containing $15.6 \mathrm{mM}$ citric acid, $89.5 \mathrm{mM}$ tribasic sodium citrate, $16.1 \mathrm{mM} \mathrm{NaH_{2 }} \mathrm{PO}_{4}$, and $128 \mathrm{mM}$ dextrose. NIH guidelines for the care and use of laboratory animals (NIH Publication no. 85-23 Rev. 1985) were observed throughout. The citrated whole blood was centrifuged at $110 \mathrm{~g}$ for $15 \mathrm{~min}$ at $22^{\circ} \mathrm{C}$. Platelet-rich plasma (PRP) was collected from the supernatant. To re-establish platelet activity, $\mathrm{CaCl}_{2}$ was added to the PRP to raise $\left[\mathrm{Ca}^{2+}\right]$ by $2 \mathrm{mM}$. Before PRP incubation, the polymercoated microtiter plate wells were prehydrated by incubating with $200 \mu \mathrm{L}$ PBS for $3 \mathrm{~h}$ at $37^{\circ} \mathrm{C}$ to achieve the optimal NO fluxes. The PBS solution was removed after this preincubation period. Then, $100 \mu \mathrm{L}$ of recalcified PRP was added to each polymer-coated well and incubated for $1 \mathrm{~h}$ at $37^{\circ} \mathrm{C}$ under static conditions. The PRP was then decanted and the wells were washed once with $200 \mu \mathrm{L}$ PBS. The entire duration from blood collection to the conclusion of the platelet adhesion step was less than $3 \mathrm{~h}$.

\section{LDH assay}

Adhered platelets were lysed using a lysing buffer which was PBS plus $1 \%(\mathrm{w} / \mathrm{v})$ Triton X-100 (Sigma) and $0.75 \%(\mathrm{w} / \mathrm{v}$ ) bovine serum albumin (Sigma). One hundred and fifty microliters of lysing buffer was incubated in each well for $1 \mathrm{~h}$ at $37^{\circ} \mathrm{C}$ with occasional agitation to completely disrupt the platelet membranes. Then, $100 \mu \mathrm{L}$ of each lysate solution was pipetted into wells of a second 96-well polystyrene microtiter plate (Fisher) that contained $100 \mu \mathrm{L}$ of reagent from an LDH assay kit (Roche Applied Sciences, Indianapolis, IN). Absorbance of each well at $490 \mathrm{~nm}$ was monitored for $1 \mathrm{~h}$ by a Labsystems Multiskan RC microplate reader (Fisher). The slopes derived from the initial 
linear portion of the absorbance vs. time curves were used for LDH quantification.

Blank experiments were performed in a similar manner. Briefly, free LDH from rabbit muscle (Roche Molecular Biochemicals, Mannheim, Germany) was dissolved in the same lysing buffer at $3 \mathrm{mU} / \mathrm{mL}$. Then, $150 \mu \mathrm{L}$ of this free $\mathrm{LDH}$ solution was placed in polymer-coated wells that had been previously hydrated with PBS buffer for $3 \mathrm{~h}$ to establish relatively stable NO release fluxes. The wells were further incubated with the $\mathrm{LDH}$ solution for $1 \mathrm{~h}$ at $37^{\circ} \mathrm{C}$. In this way, the free $\mathrm{LDH}$ would have the same duration of exposure to the same NO flux as the LDH originating from adhered platelets. The activity of the free LDH was assayed in an identical manner as the LDH in the lysate solutions.

\section{Platelet counting and LDH calibration}

Platelet rich plasma, obtained as described above, was further centrifuged at $1500 \mathrm{~g}$ for $15 \mathrm{~min}$ to produce plateletpoor plasma (PPP) and a platelet pellet. The supernatant PPP was carefully removed and the pellet of platelets was gently resuspended in Tyrode's buffer (137 $\mathrm{mM} \mathrm{NaCl}$, $95 \mathrm{mM} \mathrm{NaHCO}, 1.8 \mathrm{mM} \mathrm{CaCl}, 1.0 \mathrm{mM} \mathrm{MgCl} 2,2.7 \mathrm{mM}$ $\mathrm{KCl}, 0.4 \mathrm{mM} \mathrm{NaH} \mathrm{PO}_{4}$, and $5 \mathrm{mM}$ D-glucose). The platelet number in this suspension was counted by a Z1 Coulter particle counter (Beckman-Coulter, Miami, FL). Varying volumes of the platelet suspension derived from the pellet were also placed in the lysing buffer and incubated at $37^{\circ} \mathrm{C}$ for $1 \mathrm{~h}$ to completely release the LDH content of the platelets. Then, $100 \mu \mathrm{L}$ of each sample was mixed with equal volume of the $\mathrm{LDH}$ assay kit reagent in polystyrene microplate wells and assayed for LDH activity. The slopes obtained were used to construct the calibration curve of $\mathrm{LDH}$ activity versus platelet number, based on the Coulter counter measurement of the original stock platelet suspension in cells per milliliters.

\section{SEM analysis}

Scanning electron microscopy (SEM) images for surfaces of the various polymeric films were obtained in addition to the LDH assays. After PRP incubation, adhered platelets in some wells were fixed with $4 \%$ glutaraldehyde (Sigma) for $1 \mathrm{~h}$ and then dehydrated in a series of ethanol solutions and dried overnight. ${ }^{13}$ The bottom hemisphere was cut, sputter-coated with gold, and examined by a Hitachi S-3200N scanning electron microscope.

\section{RESULTS AND DISCUSSION}

\section{NO-releasing polymer coatings}

By using a microtiter plate with round-bottom well, a polymer coating of relatively consistent thickness can be achieved. The coating method described in the Experimental Section yields a $1.74 \mathrm{~cm}^{2}$ poly- mer film with about $60 \mu \mathrm{m}$ thickness as determined by an optical microscope. Addition of KTpCIPB to the underlying layer of the plasticized PVC film helps to achieve a more stable NO flux by maintaining charge neutrality and a constant $\mathrm{pH}$ within the polymer phase as the DBHD/ $\mathrm{N}_{2} \mathrm{O}_{2}$ species releases $\mathrm{NO}$ and creates excess amine sites in the form of DBHD. ${ }^{10}$ The NO release from DBHD/ $\mathrm{N}_{2} \mathrm{O}_{2}$ decomposition can occur via two mechanisms, protondriven $^{34}$ or thermal dissociation. ${ }^{10}$ In this experiment, temperature was maintained at $37^{\circ} \mathrm{C}$, except for the final assay of $\mathrm{LDH}$ on the microplate reader. Further, the $\mathrm{pH}$ of all solutions, including the anticoagulated PRP, was kept at $\mathrm{pH} 7.4$ for the precise manipulation of $\mathrm{NO}$ fluxes.

All of the DBHD/ $\mathrm{N}_{2} \mathrm{O}_{2}$ doped polymer films exhibited continuous NO release for over $24 \mathrm{~h}$. However, maximum $\mathrm{NO}$ fluxes were typically observed between 2 and $5 \mathrm{~h}$ after wetting and the overall magnitude of NO fluxes for the films was proportional to the amount of DBHD $/ \mathrm{N}_{2} \mathrm{O}_{2}$ doped within the underlying polymer layer of the films. The typical NO release profiles for the coatings over the first $8 \mathrm{~h}$ are shown in Figure 1(A) for films doped with $0-4$ wt \% DBHD $/ \mathrm{N}_{2} \mathrm{O}_{2}$ (and equal moles of $\mathrm{KTpClPB}$ ). It is obvious that the NO flux decays significantly for the film with the highest NO donor loading $(4 \mathrm{wt} \%)$, while the others remain relatively stable. Thus, to ensure that all platelet adhesion studies are conducted during a period of relatively constant NO flux, the time window between 3 and $4 \mathrm{~h}$ was selected for the platelet studies for all polymer film compositions examined. Figure 1(B) shows the statistics for the average flux levels at each DBHD $/ \mathrm{N}_{2} \mathrm{O}_{2}$ concentration ( $N=4$ films). Average fluxes during this period for the four different loadings were $0.93( \pm 0.07), 1.69( \pm 0.19), 3.59( \pm 0.17)$, and $7.05( \pm 0.25) \times 10^{-10} \mathrm{~mol} \mathrm{~cm}^{-2} \mathrm{~min}^{-1}$, respectively. Higher DBHD $/ \mathrm{N}_{2} \mathrm{O}_{2}$ loadings (e.g., 8 wt \%) were also tested. They yielded fluxes of over $10 \times 10^{-10}$ mol cm $\mathrm{cm}^{-2} \mathrm{~min}^{-1}$. However, the polymer surfaces become much rougher in this case, as determined by SEM. This is likely due to the poor solubility of $\mathrm{DBHD} / \mathrm{N}_{2} \mathrm{O}_{2}$ in the polymer matrix and therefore the surface roughness becomes more pronounced as the DBHD $/ \mathrm{N}_{2} \mathrm{O}_{2}$ concentration goes above 4 wt $\%$.

\section{Platelet adhesion}

Unlike radioactive labeling of platelet, the major advantage of the LDH assay is its simplicity which minimizes possible activation of resting platelets. Hence it has the potential to be more reproducible. This attribute is critical for the evaluation of potentially more biocompatible materials. Platelet adhesion experiments were repeated using three different 
(A)

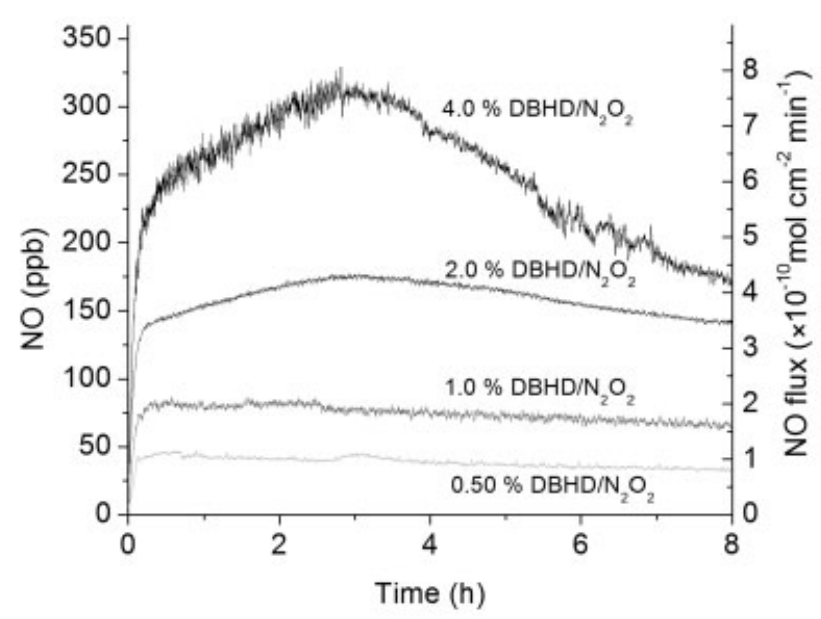

(B)

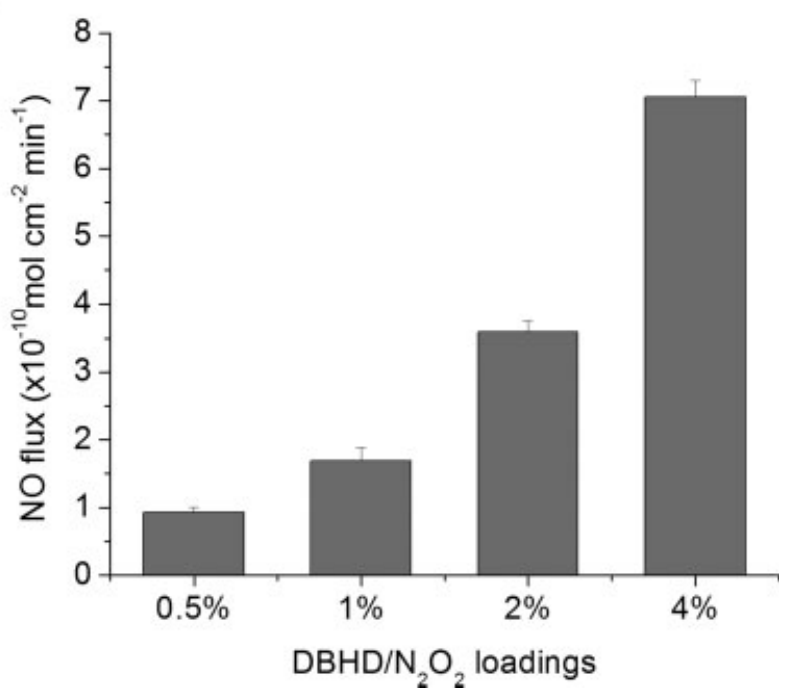

Figure 1. A: NO surface flux profiles for polymer-coated wells doped with 0.50, 1.0, 2.0, and 4.0 wt $\%$ DBHD/ $\mathrm{N}_{2} \mathrm{O}_{2}$ and equimolar $\mathrm{KTpClPB}$. Wells were bathed in $\mathrm{PBS}$ at $37^{\circ} \mathrm{C}$ as measured by chemiluminescence NO analyzer. B: Average NO fluxes and SD during the 3-4 h time window for each DBHD/ $\mathrm{N}_{2} \mathrm{O}_{2}$ loading. $N=4$ for each loading.

lots of blood (from different animals) and the results were found to be quite reproducible. During the initial incubation step, PRP was in contact with $0.834 \mathrm{~cm}^{2}$ of polymer surface in each well. This surface area was thus used to calculate the average platelet density on the films after determining LDH activity. However, it should be noted that the platelet coverage was not even over the entire surface of the polymer films (as determined by SEM), likely owing to the hemispherical shape of the wells. No fixation was used after platelet adhesion since platelets fixed with the common fixative, glutaraldehyde, exhibited no LDH activity in the subsequent assay. Therefore, the unfixed platelets were loosely adhered to the polymer surfaces. Rinsing the wells three times yielded near-baseline LDH activities across all polymers tested, probably because of the removal of adhered platelets. In the final protocol, the wells were washed only once after platelet incubation, which was found to be sufficient to distinguish the extent of platelet adhesion as a function of NO flux.

\section{LDH assay}

Prior to testing the effect of NO with respect to platelet adhesion, it was necessary to establish that NO has no effect on LDH activity. As a free radical, $\mathrm{NO}$ is known to react with various enzymes, which could possibly change their function and efficacy in catalysis. $^{35,36}$ Previous studies using the LDH assay to assess the biocompatibility of polymers involved evaluating only physically ${ }^{30,32}$ or chemically ${ }^{29,31}$ modified surfaces, not a material that releases a reactive species such as NO. Consequently, it was crucial to verify that any "observed" reduction in LDH activity derived from experiments using PRP was indeed the results of less adhered platelets rather than any inhibition of LDH function in the presence of continuous NO production.

An appropriate blank experiment was performed to evaluate the influence of continuous $\mathrm{NO}$ release on LDH activity. The enzyme concentration was set at $3 \mathrm{mU} / \mathrm{mL}$ since this was the level typically seen for the control experiments (after cell lysis) with platelets.

Table I lists the free LDH activity in the control and DBHD $/ \mathrm{N}_{2} \mathrm{O}_{2}$ loaded wells, as well as the blank with only plain 1:1 PVC/DOS films and no other additives. Interestingly, the control wells have slightly lower apparent LDH activities. LDH activity also decreases with higher NO donor loading in the polymer films. Since $\mathrm{LDH}$ has an isoelectric $\mathrm{pH}$ value (pI) of 4.6, it will be negatively charged in the PBS buffer. Thus, electrostatic interaction with the positively-charged surface of the polymers is a possible explanation for the data shown in Table I. The control polymer has the highest concentration (4 wt \%)

TABLE I

Free LDH Activity After 1-h Incubation in Blank (Plain PVC/DOS), Control, and Various NO-Releasing Polymer-Coated Wells, as Determined by Colorimetric Reaction Rate

\begin{tabular}{lrcrrrr}
\hline Composition & Blank & Control & $0.5 \%$ & $1 \%$ & $2 \%$ & $4 \%$ \\
\hline \% LDH & 100.0 & 89.7 & 103.6 & 98.1 & 98.9 & 93.3 \\
SD & 0.2 & 3.3 & 2.4 & 2.7 & 2.0 & 0.9
\end{tabular}

LDH activity was set to $100 \%$ in blank polymer wells and others were plotted as the relative percentage.

$N=3$ for each polymer composition. 
of free amine (DBHD) which will gradually become protonated to create a positively charged surface (as it diffuses into the top coat layer of plain PVC polymer). Once LDH is attracted to polymer surface, less free $\mathrm{LDH}$ will be measured in the bulk solution, hence giving a false-negative result. In the case of the NO-releasing polymers, the amine will also be formed upon hydrolysis of the diazeniumdiolate. ${ }^{34}$ However, at the time of assay, the diazeniumdiolate is not $100 \%$ hydrolyzed and therefore amine sites in NO-releasing polymers will be fewer compared to those in the control. Because of this difference in amine site density between the diazeniumdiolated and nondiazeniumdiolated DBHD, the false negatives cannot be simply corrected by simultaneously collecting the LDH activities from same weight percent control and DBHD $/ \mathrm{N}_{2} \mathrm{O}_{2}$ loaded polymer films. Even though a top coat was applied and bovine serum albumin was added to the lysing buffer to minimize nonspecific adsorption of LDH to the surface of the polymers, it cannot be completely eliminated. This false-negative result from the control polymers suggested that the actual LDH levels in control polymer coated wells could be $\sim 10 \%$ higher than that measured from this LDH assay (see below).

Basal levels of LDH present in plasma could also adsorb onto the polymers and contribute to a common baseline across all polymers tested. It has been reported that PRP contains significant LDH activity and that LDH activity can be detected on polymer surfaces incubated with PPP. ${ }^{28}$ This adsorbed LDH may later be desorbed during the cell lysing stage and contribute to total LDH activity in this assay. To prove this, PRP was replaced by PPP in the platelet adhesion step, followed by identical washing, lysis, and assay procedures. The baseline LDH level in PPP was found to be equivalent to a platelet density of about $1.00( \pm 0.25) \times 10^{5} \mathrm{~cm}^{-2}$ on all polymers. This baseline was not subtracted from the platelet adhesion results, since there could be more cytolysis in a given preparation of PPP (as it was prepared by centrifugation at a much higher RCF than PRP) that can give rise to a higher serum LDH level. However, the presence of this background LDH activity derived from the plasma suggests an even greater reduction, percentage-wise, of adhered platelets on NO-releasing polymers for the experiments described below.

As shown in Figure 2, fewer platelets adhered on polymer surfaces as the NO flux increases. The control polymer has an apparent platelet density of 14.0 $( \pm 2.5) \times 10^{5} \mathrm{~cm}^{-2}$, while the $0.5,1,2$, and $4 \%$ DBHD $/ \mathrm{N}_{2} \mathrm{O}_{2}$ doped films reduced the platelet density to $7.06( \pm 1.0), 4.82( \pm 0.79), 4.16( \pm 0.68)$, and $2.96( \pm 0.21) \times 10^{5} \mathrm{~cm}^{-2}$, respectively $(N=12$ for all experiments, using at least three different blood lots as the source for the PRP). In all cases, platelet adhe-

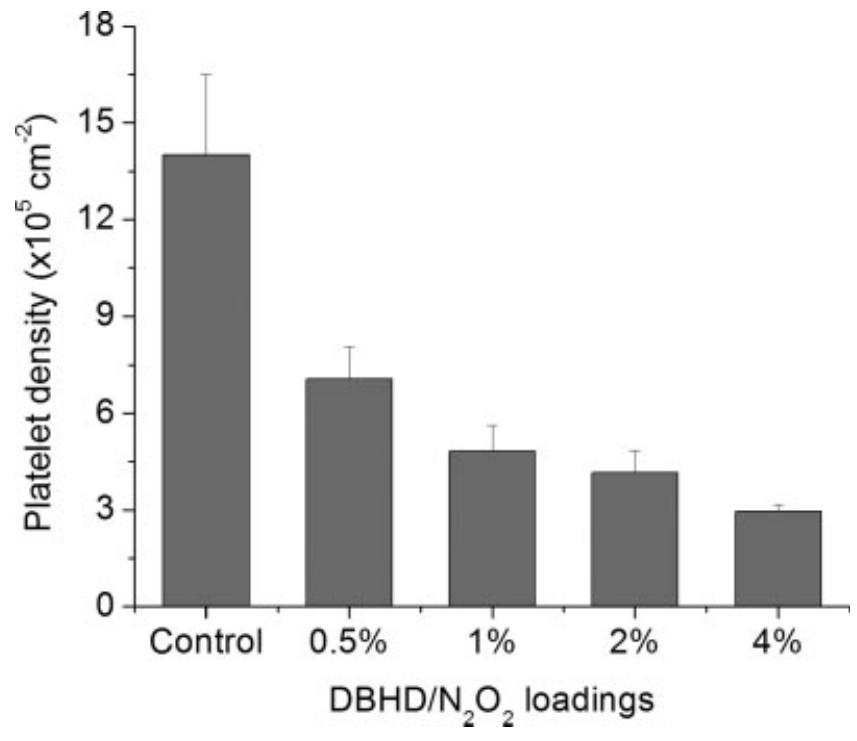

Figure 2. Platelet adhesion density on control and various DBHD $/ \mathrm{N}_{2} \mathrm{O}_{2}$ loaded polymer coatings as determined by LDH assay. $N=12$ for each polymer composition.

sion on the NO-releasing polymers is statistically different from the control $(p<0.0001)$. The influence of LDH adsorption via the mechanisms discussed above is insignificant as compared to the greater reduction of LDH activities on NO-releasing polymer surfaces. Further, the NO dose dependence on platelet adhesion appears to be nonlinear (see Fig. 2), with the largest percent change in platelet adhesion occurring for the $0.5 \mathrm{wt} \%$ of DBHD/ $\mathrm{N}_{2} \mathrm{O}_{2}$ doped film which provides the lowest NO flux. This observation is consistent with the nonlinear dose responses seen in previous studies that examined platelet adhesion in the presence of varying levels of NO or NO donors. ${ }^{6-9}$

Polymers with higher DBHD/ $\mathrm{N}_{2} \mathrm{O}_{2}$ loadings (i.e. 8,12 , and $16 \mathrm{wt} \%$ ) have also been tested in the above manner. Given that DBHD $/ \mathrm{N}_{2} \mathrm{O}_{2}$ has poor solubility in these polymer matrices, it is not surprising that more platelet adhesion occurs on these rougher surfaces despite the fact that the NO fluxes are even higher. Indeed, another in vivo model with DBHD $/ \mathrm{N}_{2} \mathrm{O}_{2}$ coated on the inner walls of rabbit extracorporeal circuits provided similar resultswith an intermediate level of $\mathrm{DBHD} / \mathrm{N}_{2} \mathrm{O}_{2}$ loading yielding the best biocompatibility (unpublished results). However, such results do not necessarily suggest the inability of NO to inhibit platelet adhesion and activation at those higher fluxes. Should a new lipophilic NO donor be developed that can provide smooth surfaces while still maintaining the desirable properties of $\mathrm{DBHD} / \mathrm{N}_{2} \mathrm{O}_{2}$ (e.g., minimal donor leaching and wide range of attainable $\mathrm{NO}$ fluxes), the effect of platelet inhibition by NO fluxes higher than those tested herein could be explored in more detail. 


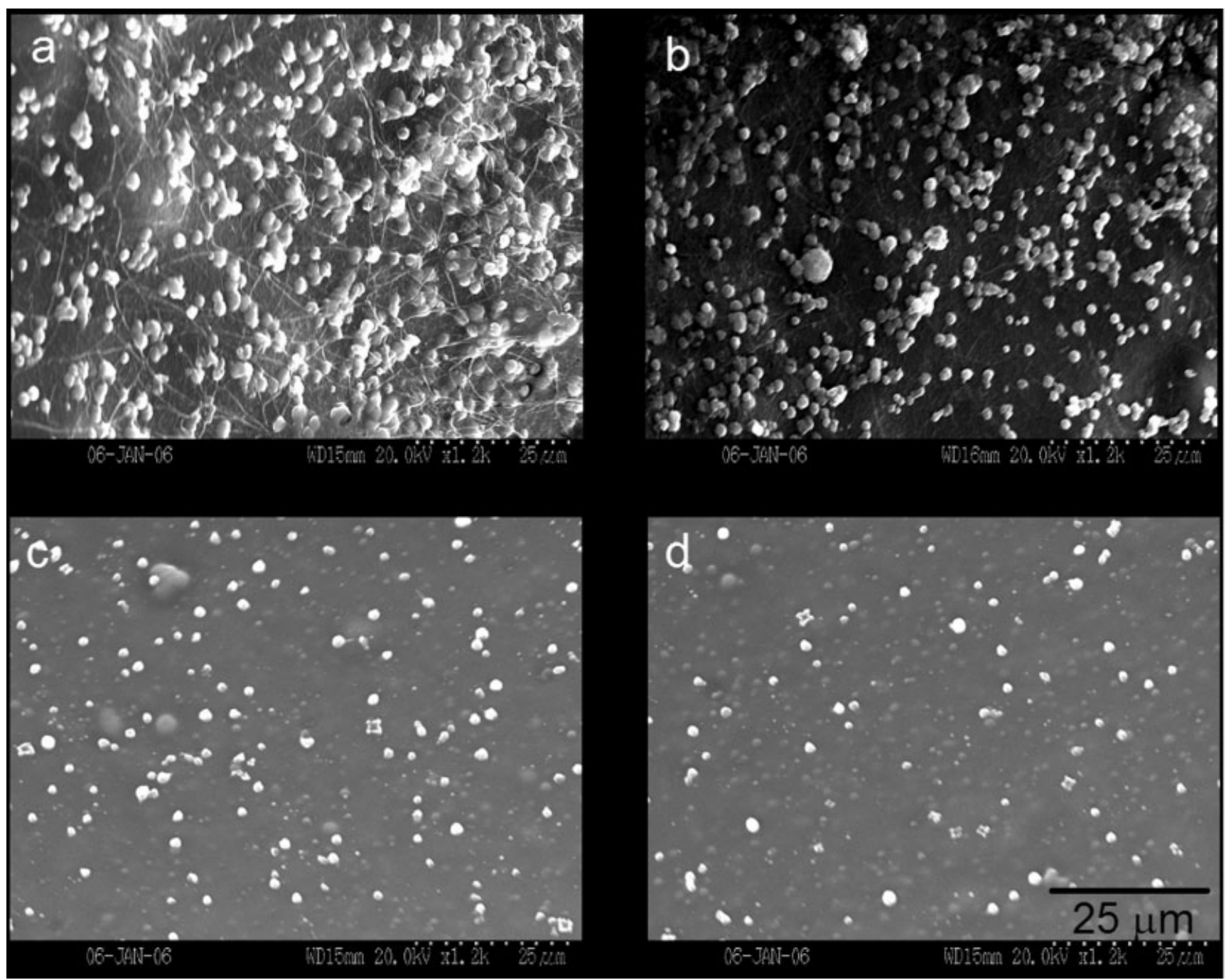

Figure 3. SEM micrographs of adhered platelets on control polymers (a and b) and 4 wt $\%$ DBHD/ $\mathrm{N}_{2} \mathrm{O}_{2}$ loaded polymers (c and d) after $1 \mathrm{~h} \mathrm{PRP} \mathrm{incubation.}$

\section{SEM analysis}

To support LDH assay, SEM images were taken from the control and $4 \% \mathrm{DBHD} / \mathrm{N}_{2} \mathrm{O}_{2}$ doped films. The SEM micrographs shown in Figure 3 were taken at the center of the well bottoms. It is obvious that platelets on NO-releasing surfaces were much less activated compared with those on the control surface. Also, there were much fewer platelets per viewing area on NO-releasing surfaces. However, SEM does not serve as a quantitative measure since the surface it examined constitutes only a tiny portion of the total surface in contact with PRP. This is the reason why LDH assay was used to evaluate average platelet adhesion over the entire surfaces.

\section{CONCLUSION}

The thromboresistance of healthy endothelium is a complex, synergistic mechanism in which NO plays a very important role. In this article, an in vitro platelet adhesion assay via platelet $\mathrm{LDH}$ release is described to study the hemocompatibility of polymer films that continuously release small fluxes of $\mathrm{NO}$, chemically, to mimic the function of human endothelium. Using this LDH assay, it was found that continuous NO release from 1:1 PVC/DOS polymer film matrices at close to physiological fluxes $\left(0.93 \times 10^{-10}\right.$ to $7.05 \times 10^{-10} \mathrm{~mol} \mathrm{~cm}^{-2} \mathrm{~min}^{-1}$ ) helps to reduce platelet adhesion from $14.0( \pm 2.1) \times 10^{5}$ to 2.96 $( \pm 0.18) \times 10^{5}$ cells $\mathrm{cm}^{-2}$ on such polymer surfaces. SEM micrographs support the findings with the LDH assay by showing that the platelets were fewer in number and less activated on the NO-releasing surfaces. Accuracy of this assay is somewhat affected by the adsorption of LDH on polymer surfaces. Because of electrostatic interactions between the positively-charged polymer surface and the negativelycharged LDH, the actual adhered platelets on polymers with the highest $\mathrm{NO}$ flux could be $\sim 7 \%$ more 
than that measured in this assay. However, this false-negative is more significant $(\sim 10 \%)$ with control polymers which have the highest positive charge. The basal LDH level present in plasma is likely to contribute to a common baseline for all polymers tested, a value that is difficult to precisely determine. Nevertheless, taking such background levels into consideration only strengthens our finding that the LDH assay scheme can be employed to study, in vitro, the effect of NO release on platelet adhesion.

It should be noted that the fluxes determined to reduce platelet adhesion for the in vitro studies described above may be different than the fluxes required to obtain a similar decrease in platelet adhesion when the same polymer surfaces are in contact with flowing whole blood. This is because the surface concentrations of $\mathrm{NO}$ gas will vary depending on the composition of the flowing blood, including scavenging reactions with high levels of endogenous hemoglobin in red blood cells, thiol groups, and normal oxidation of NO by blood oxygen levels. In addition, platelet adhesion will also be influenced by hemodynamics. ${ }^{37}$ Clearly, the methodology reported here using microtiter plate wells coated with polymer that release NO only provides a preliminary assessment as to the potential capability of such coatings to prevent thrombosis when used in vivo. Ultimately, the LDH assay methodology described herein should also be used to assess platelet adhesion on NO-releasing materials that are tested intravascularly. Such studies are currently in progress in this laboratory.

We thank Amy Skrzypchak and Nathan Lafayette in the Extracorporeal Membrane Oxygenation (ECMO) lab at University of Michigan medical school for preparing the anticoagulated rabbit blood.

\section{References}

1. Feldman PL, Griffith OW, Stuehr DJ. The surprising life of nitric-oxide. Chem Eng News 1993;71:26-38.

2. Diodati JG, Quyyumi AA, Hussain N, Keefer LK. Complexes of nitric oxide with nucleophiles as agents for the controlled biological release of nitric oxide-Antiplatelet effect. Thromb Haemost 1993;70:654-658.

3. Maragos CM, Morley D, Wink DA, Dunams TM, Saavedra JE, Hoffman A, Bove AA, Isaac L, Hrabie JA, Keefer LK. Complexes of NO with nucleophiles as agents for the controlled biological release of nitric oxide-Vasorelaxant effects. J Med Chem 1991;34:3242-3247.

4. Vaughn MW, Kuo L, Liao JC. Estimation of nitric oxide production and reaction rates in tissue by use of a mathematical model. Am J Physiol Heart Circ Physiol 1998;43:H2163-H2176.

5. Michiels C. Endothelial cell functions. J Cell Physiol 2003;196: 430-443.

6. Hernandez MR, Tonda R, Arderiu G, Pino M, Serradell M, Escolar G. Antithrombotic effect of a new nitric oxide donor (LA419) on experimental thrombogenesis. Eur J Clin Invest 2005;35:337-342.
7. Masters KSB, Lipke EA, Rice EEH, Liel MS, Myler HA, Zygourakis C, Tulis DA, West JL. Nitric oxide-generating hydrogels inhibit neointima formation. J Biomater Sci Polym Ed 2005;16:659-672.

8. Rossiello MR, Momi S, Caracchini R, Giannini S, Guglielmini G, Monopoli A, Ongini E, Semeraro N, Colucci M, Gresele P. A novel nitric oxide-releasing statin derivative exerts an antiplatelet/antithrombotic activity and inhibits tissue factor expression. J Thromb Haemost 2005;3:2554-2562.

9. Ramamurthi A, Lewis RS. Design of a novel apparatus to study nitric oxide (NO) inhibition of platelet adhesion. Ann Biomed Eng 1998;26:1036-1043.

10. Batchelor MM, Reoma SL, Fleser PS, Nuthakki VK, Callahan RE, Shanley CJ, Politis JK, Elmore J, Merz SI, Meyerhoff ME. More lipophilic dialkyldiamine-based diazeniumdiolates: Synthesis, characterization, and application in preparing thromboresistant nitric oxide release polymeric coatings. J Med Chem. 2003;46:5153-5161.

11. Zhang HP, Annich GM, Miskulin J, Osterholzer K, Merz SI, Bartlett RH, Meyerhoff ME. Nitric oxide releasing silicone rubbers with improved blood compatibility: Preparation, characterization, and in vivo evaluation. Biomaterials 2002;23:1485-1494.

12. Parzuchowski PG, Frost MC, Meyerhoff ME. Synthesis and characterization of polymethacrylate-based nitric oxide donors. J Am Chem Soc 2002;124:12182-12191.

13. Frost MC, Rudich SM, Zhang HP, Maraschio MA, Meyerhoff ME. In vivo biocompatibility and analytical performance of intravascular amperometric oxygen sensors prepared with improved nitric oxide-releasing silicone rubber coating. Anal Chem 2002;74:5942-5947. Erratum: 2003;75:1037.

14. Schoenfisch MH, Mowery KA, Rader MV, Baliga N, Wahr JA, Meyerhoff ME. Improving the thromboresistivity of chemical sensors via nitric oxide release: Fabrication and in vivo evaluation of NO-releasing oxygen-sensing catheters. Anal Chem 2000;72:1119-1126.

15. Mowery KA, Schoenfisch MH, Saavedra JE, Keefer LK, Meyerhoff ME. Preparation and characterization of hydrophobic polymeric films that are thromboresistant via nitric oxide release. Biomaterials 2000;21:9-21.

16. Espadas-Torre C, Oklejas V, Mowery K, Meyerhoff ME. Thromboresistant chemical sensors using combined nitric oxide release ion sensing polymeric films. J Am Chem Soc 1997;119:2321-2322.

17. Zhang HP, Annich GM, Miskulin J, Stankiewicz K, Osterholzer K, Merz SI, Bartlett RH, Meyerhoff ME. Nitric oxide-releasing fumed silica particles: Synthesis, characterization, and biomedical application. J Am Chem Soc 2003;125:5015-5024.

18. Zhou ZR, Meyerhoff ME. Polymethacrylate-based nitric oxide donors with pendant $\mathrm{N}$-diazeniumdiolated alkyldiamine moieties: Synthesis, characterization, and preparation of nitric oxide releasing polymeric coatings. Biomacromolecules 2005;6:780-789.

19. Zhou ZR, Meyerhoff ME. Preparation and characterization of polymeric coatings with combined nitric oxide release and immobilized active heparin. Biomaterials 2005;26:6506-6517.

20. Reynolds MM, Zhou ZR, Oh BK, Meyerhoff ME. Bis-diazeniumdiolates of dialkyldiamines: Enhanced nitric oxide loading of parent diamines. Org Lett 2005;7:2813-2816.

21. Bohl KS, West JL. Nitric oxide-generating polymers reduce platelet adhesion and smooth muscle cell proliferation. Biomaterials 2000;21:2273-2278.

22. Smith DJ, Chakravarthy D, Pulfer S, Simmons ML, Hrabie JA, Citro ML, Saavedra JE, Davies KM, Hutsell TC, Mooradian DL, Hanson SR, Keefer LK. Nitric oxide-releasing polymers containing the N(O)NO (-) group. J Med Chem 1996;39:1148-1156.

23. Wong $\mathrm{K}, \mathrm{Li} \mathrm{XB}$. Nitric oxide infusion alleviates cellular activation during preparation, leukofiltration and storage of platelets. Transfus Apher Sci 2004;30:29-39.

24. Shahbazi T, Jones N, Radomski MW, Moro MA, Gingell D. Nitric-oxide donors inhibit platelet spreading on surfaces coated 
with fibrinogen but not with fibronectin. Thromb Res 1994;75: 631-642

25. Whiss PA, Andersson RGG, Srinivas U. Modulation of P-selectin expression on isolated human platelets by an NO donor assessed by a novel ELISA application. J Immunol Methods 1997;200(1/2):135-143.

26. White JG. Electron microscopy methods for studying platelet structure and function. In: Gibbins JM, Mahaut-Smith MP, editors. Methods in Molecular Biology (Platelets and Megakaryocytes). Totowa, NJ: Humana; 2004. pp 47-63.

27. Rodrigues $\mathrm{M}$, Sinzinger $\mathrm{H}$. Platelet labeling-Methodology and clinical-applications. Thromb Res 1994;76:399-432.

28. Tamada Y, Kulik EA, Ikada Y. Simple method for platelet counting. Biomaterials 1995;16:259-261.

29. Tsai WB, Grunkemeier JM, Horbett TA. Human plasma fibrinogen adsorption and platelet adhesion to polystyrene. J Biomed Mater Res 1999:44:130-139.

30. Park JY, Gemmell CH, Davies JE. Platelet interactions with titanium: Modulation of platelet activity by surface topography. Biomaterials 2001;22:2671-2682.

31. Suggs LJ, West JL, Mikos AG. Platelet adhesion on a bioresorbable poly(propylene fumarate-co-ethylene glycol) copolymer. Biomaterials 1999;20:683-690.
32. Kikuchi L, Park JY, Victor C, Davies JE. Platelet interactions with calcium-phosphate-coated surfaces. Biomaterials 2005;26: 5285-5295.

33. Rivera J, Lozano ML, Vicente V. In vitro changes of platelet parameters: Lessons from blood blanking. In: Gibbins JM, MahautSmith MP, editors. Methods in Molecular Biology (Platelets and Megakaryocytes). Totowa, NJ: Humana; 2004. pp 57-72.

34. Davies KM, Wink DA, Saavedra JE, Keefer LK. Chemistry of the diazeniumdiolates, Part 2. Kinetics and mechanism of dissociation to nitric oxide in aqueous solution. J Am Chem Soc 2001;123:5473-5481.

35. Wassmann S, Wassmann K, Nickenig G. Modulation of oxidant and antioxidant enzyme expression and function in vascular cells. Hypertension 2004;44:381-386.

36. Kwiecien I, Sokolowska M, Luchter-Wasylewska E, Wlodek L. Inhibition of the catalytic activity of rhodanese by S-nitrosylation using nitric oxide donors. Int J Biochem Cell Biol 2003;35: 1645-1657.

37. Dejana E, Remuzzi A, Languino LR, Costantini V, Lauri D, Zanetti A, Degaetano G. Human-platelet adhesion to subendothelium under controlled hemodynamic conditions-A methodological approach. Methods Find Exp Clin Pharmacol 1985;7: 153-159. 